\title{
Spatially Resolved Improved FT-IR Microspectroscopy of Deuterated Species in Tissue
}

\author{
David L. Wetzel ${ }^{1}$ and Steven M. LeVine ${ }^{2}$ \\ ${ }^{1}$ Microbeam Molecular Spectroscopy Laboratory, Kansas State University, Manhattan, KS \\ 66506, ${ }^{2}$ University of Kansas Medical Center, Dept. of Molecular \& Integrative Physiology, \\ 3901 Rainbow Blvd., Kansas City, KS 66160, U. S. A.
}

\section{Introduction:}

Stretching vibrations of $\mathrm{CH}, \mathrm{NH}$, and $\mathrm{OH}$ observable with FT-IR microspectroscopy have allowed a means of localizing compounds containing deuterium isotopes in plant and mammalian tissue. Relatively high detection limits heretofore have required thicker than usual sections or a sufficient quantity of deuterated compound applied or accumulated in the tissue to allow detection and reliable peak area determination. This paper is concerned with spectra obtained with a recently improved instrumental capability that has reduced noise and increased detector sensitivity in the $2600-2000 \mathrm{~cm}^{-1}$ region of the spectrum.

\section{Previous Work:}

The migration of water in wheat kernels during the tempering process prior to flour milling was studied. Wheat kernels soaked in $\mathrm{D}_{2} \mathrm{O}$ were withdrawn at different time intervals, frozen, sectioned, placed between barium flouride windows, and immediately line mapped to trace the movement of the tempering heavy water in the presence of natural water already in the seed $[1,2]$. Twenty micrometer thick sections gave measurable OD peak areas while other absorption bands were off scale. Metabolically incorporated CD and ND/OH deposits in the cerebella of adult rats that had $30-40 \% \mathrm{D}_{2} \mathrm{O}$ in their drinking water for $5 \frac{1}{2}$ weeks were examined. A multitude of spectra from line mapping across layers of white matter, nuclear cell layer, and molecular cell layer showed the deposition of the CD and combined ND, OD bands for each of the three different layers. The hydrophobic white matter had the greatest amount of CD and hydrophilic nuclear cell layers had the greatest amount of the other deuterated forms. The potential for the study of brain metabolism was suggested by comparison of the peak area of CD with $\mathrm{CH}$ etc. to reveal uptake of the deuterium isotope from the ingested $\mathrm{D}_{2} \mathrm{O}$ [3]. The spot size analyzed with a synchrotron illuminated infrared microspectroscope was $12 \Phi \mathrm{m}$ x $12 \Phi \mathrm{m}$. Additional spectra were collected using an infrared microscope with a conventional globar source from $36 \Phi \mathrm{m}$ x $36 \Phi \mathrm{m}$ spot areas. These latter data were used for statistical treatment.

\section{Preliminary Observations and Application of the Current Instrumental Capability:}

Intermediate to the work reported in the past with the wheat kernels and rat cerebella preliminary experiments with deuterated organic compounds and animal tissue with a conventional globar source and thin, $8 \Phi \mathrm{m}$, sections showed the need for lower detection limits. Several instrument modifications were made and further experimentations obtained spectra from neat deuterated organic compounds as well as the same compounds on a tissue substrate. Also, deuterated compounds that were applied topically or systemically were found subsequently by observation of the CD absorption band detected above baseline noise in tissue from the experimental animals. These data indicated the potential of the microspectroscopic technique with improved detection limits. The instrument in current use is a Continu $\Phi \mathrm{m}^{\circledR}$ equipped with a 
custom detector that has the single beam response shown in figure 1A superimposed on a tissue spectrum 1B. Details of this custom made detector, its specifications and testing in comparison to a conventional MCT detector have been previously described [4]. Not only is the detector more sensitive at the wavelength of concern, but a significant benefit is derived from the fact that it is relatively insensitive at other wavelengths thus reducing the total noise. The system described is now in routine use on a number of animal experiments. Several of these involve layered tissues that vary in their chemical composition and physiological function. The ability to use the deuterated compounds enables tracing experiments to investigate their deposition or their disappearance from localized spatially resolved areas in the tissue. Figure 2 shows differences in the CD bands obtained at different layers of the same tissue indicative of laalized population corresponding to the region indicated by the arrows in figure 1B. Other examples of recent and current investigations are included in the presentation.
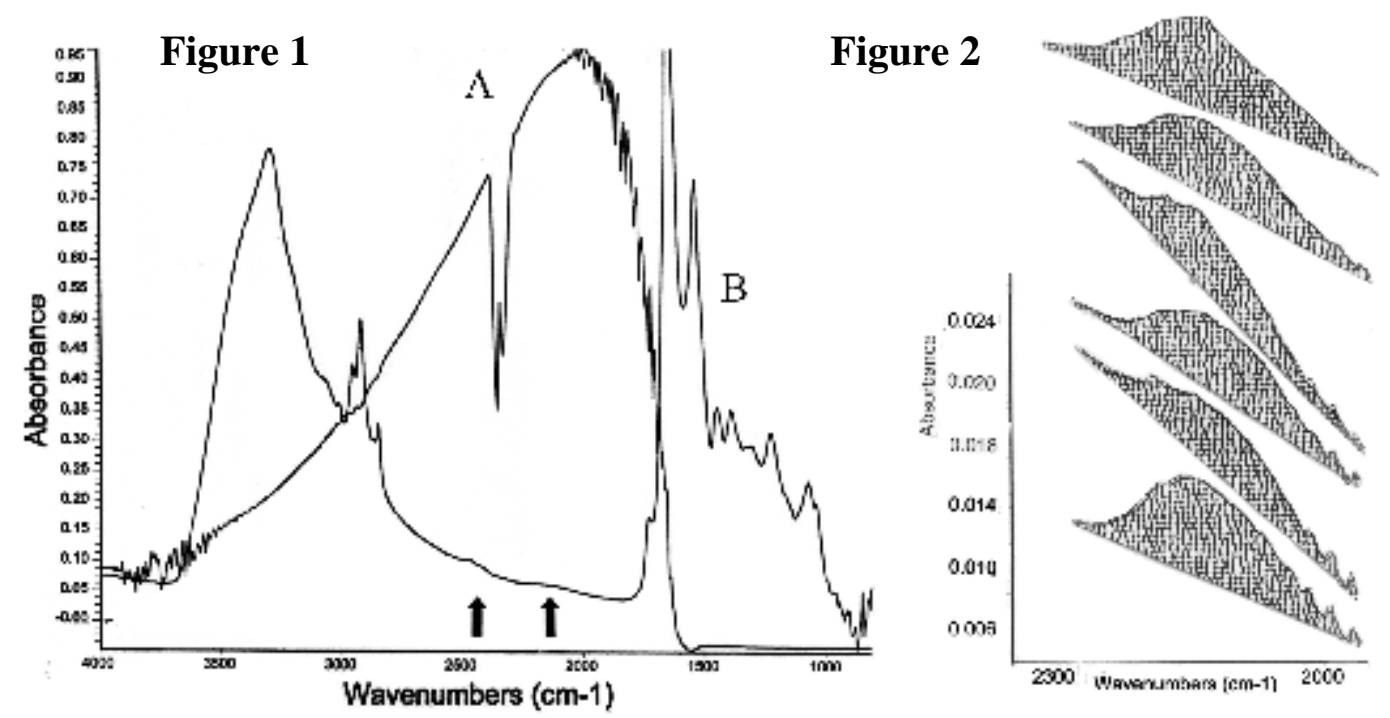

\section{Summary:}

Reduced detection limits enable peak area determination to be made from spatially resolved FT-IR microspectroscopic data from sections of conventional thickness on a routine basis to enhance the metabolic studies.

\section{References}

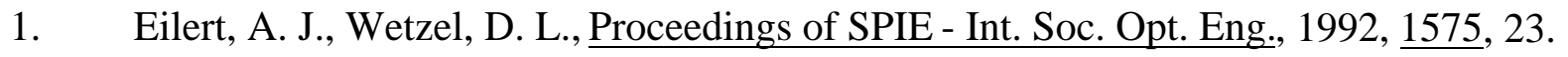

2. Sweat, J. A., "Vibrations in Tempering Times for Winter Wheats by IR Microspectroscopic Tracking of $\mathrm{D}_{2} \mathrm{O}$ ”, MS, Thesis, Kansas State University, 1996.

3. Wetzel, D. L., Slatkin, D.N., LeVine, S. M., Cell. Mol. Biol., 1998, 44(1), 15-27.

4. Wetzel, D. L., "Sensitive Infrared Narrow Band Optimized Microspectrometer", $1^{\text {st }}$ Int. Conf. Adv. Vibrational Spectroscopy, Turku, Finland, August 2001, no. 115. 